\title{
Transmission of Baby's Heartbeat Rhythm to Its Parent Via Internet and Vice Versa*
}

\author{
Takeshi KAWASHIMA ** \\ ** Department of Mechanical Engineering, Kanagawa Institute of Technology, \\ 1030 Shimo-ogino, Atsugi-shi, Kanagawa 243-0292, Japan \\ E-mail: kawashima@eng.kanagawa-it.ac.jp
}

\begin{abstract}
In Japan, the declining birthrate has resulted in a social problem, namely, the shortage of a young labor force. One of the ways by which this issue can be resolved is by establishing social surroundings in which parents with a baby are able to work comfortably. In this study, a system is proposed that can give comfort and peace of mind to a baby by transmitting the heartbeat rhythm of its parent to the baby through the Internet and simulating the transmitted heartbeat rhythm in a doll held by the baby or in a pillow. Moreover, the system also gives peace of mind to the parent by transmitting the baby's heartbeat rhythm to the parent and simulating the transmitted rhythm by means of a portable simulator like a wristwatch. An evaluation experiment is carried out to confirm whether a baby responds well to the heartbeat rhythm of its mother, which is simulated in a pillow, and that the mother can recognize the heartbeat rhythm of her baby by using the portable heartbeat simulator. The results revealed that when a baby is interested in the simulated sound, it prefers the simulated heartbeat rhythm of its mother rather than an artificial heartbeat rhythm with a constant period and the condition that the stimulation stops. Therefore, it is confirmed that the proposed system can give peace of mind to both a baby and its parent.
\end{abstract}

Key words: Human Engineering, Heartbeat Rhythm, Comfortable Rhythm, Heartbeat Transmission, Fluctuation of Heartbeat, Internet, LAN, Parent, Infant, Heartbeat Simulator

\section{Introduction}

In modern Japan, the time for which a baby is separated from its parents has increased as the number of dual-career families has increased due to the social progression of women. On the other hand, a woman who seeks to pursue a career must retire or cannot resume her work for some time because she must take care of her baby. This situation has led to a decrease in the birthrate and a shortage of young labor force. These are serious social problems. Therefore, in order to solve the above problems, it is necessary to establish social surroundings in which parents with a baby can work comfortably. In particular, one of the problems in Japan is that working parents cannot take care of their babies from the morning to the evening when they go to work. This is because the time taken to commute to one's workplace and the psychological distance from one's home to workplace is perceived as being very long due to heavy traffic and commuter rush, although the physical distance between the house and the workplace is not long. In this study, a system is proposed for shortening the psychological distance between a parent working at the workplace and his/her baby left at its grandparents' home or at a nursery. 
In a mother's womb, a fetus grows sensing the heartbeats of the mother. Therefore, it is considered that a baby feels relaxed on sensing the heartbeat of its mother. For example, a crying baby is pacified when it is held by its mother. In this situation, a large factor in pacifying the baby seems to be the sense of the mother's heartbeats to which the baby is accustomed, although the stableness, the smell, the skin warmth, the swinging and holding actions, etc. also contribute to pacifying the baby. Therefore, in the proposed system, the parent's heartbeat rhythm is transmitted to the baby through the ubiquitous Internet and simulated the rhythm in a doll held by the baby or in a pillow so that the baby senses the existence of its parent. Simultaneously, this system enables the parent to sense the existence of the baby by transmitting the baby's heartbeat rhythm to the parent through the Internet. Further, in order to confirm that the proposed system can give peace of mind to both a baby and its parent, an evaluation experiment is carried out. In the experiment, it is examined whether a baby responds well to the mother's heartbeat rhythm simulated in a pillow. It is also confirmed whether the mother is able to recognize her baby's heartbeat rhythm as simulated by a portable heartbeat simulator.

In order to verify that the heartbeat rhythm does simulate a pleasant situation, a unique study was conducted by the present author; in that study, an active rocking chair driven by a solenoid was controlled by synchronizing the chair motion with the heartbeat rhythm of the seated person ${ }^{1)}$. Through an evaluation experiment, it was clarified that the power spectrum of the envelope of the acceleration waveform measured near the ear of the seated person exhibited an approximately $1 / \mathrm{f}$ profile, which generally appears when a person feels comfortable.

\section{System for Internet Transmission of Heartbeat Rhythm}

Figure 1 shows an illustration of the system used for the Internet transmission of the heartbeat rhythm. The heartbeat of a parent is measured using a pulse sensor, and the measured signal is amplified and converted into a digital TTL signal by means of a heartbeat detector. In this study, an ear sensor resembling a small clothespin, which is worn on an earlobe, is selected as the pulse sensor because it does not cause much discomfort to the parent. Such an ear sensor is generally used with an exercise bicycle (or an ergometer) in health clubs. Further, the heartbeat detector converts the measured signal by using a differentiator in order to simulate the pressure sensation by the movement of the heart as the infant set the face to the breast of the mother. Figure 2 shows a photograph of the ear sensor and the heartbeat detector that has a size of $90 \mathrm{~mm}$ (length) $\times 56 \mathrm{~mm}$ (width). A clip is attached to the wires of the sensor so that the wires can be positioned conveniently, say, on the collar, etc. The measured waveforms of the signal converted in the heartbeat detector are shown in Fig. 3. The amplified ear sensor signal is presented by the bold red line, the converted signal by the differentiator by the solid yellow line, and the output TTL signal by the broken blue line. From this figure, it is clarified that the heartbeat detector outputs $5 \mathrm{~V}$ when the blood is pumped and $0 \mathrm{~V}$ when the blood is not pumped. The converted TTL signal is transmitted to a single-board microcomputer having a LAN interface with a size of $156 \mathrm{~mm}$ (length) $\times 114 \mathrm{~mm}$ (width), and times during the TTL signal is ON and OFF are measured. The times are transmitted to another single-board microcomputer as the numerical character data and reconverted to the original TTL signal. Then, the TTL signal is transmitted to a heartbeat rhythm simulator by a radio using a small wireless transmitter and receiver with a size of $40 \mathrm{~mm}$ (length) $\times 20 \mathrm{~mm}$ (width). Figure 4 shows the single-board microcomputer having the LAN interface and the wireless transmitter installed on it. The heartbeat rhythm simulator installed in a pillow generates stimulation that simulates the heartbeat rhythm by using a solenoid that pushes against a thin plastic plate covered by rubber. Figure 5 shows the heartbeat rhythm simulator operated by three dry cell batteries of 
$9 \mathrm{~V}(006 \mathrm{P})$. Figure 6 shows the pillow with the built-in heartbeat rhythm simulator. Figure 7 shows the waveform of the TTL signal transmitted to the simulator presented by the broken blue line and the time history of the solenoid displacement by the bold pink line. From this figure, it is clarified that the simulator creates the pressure sensation as the blood is pumped.

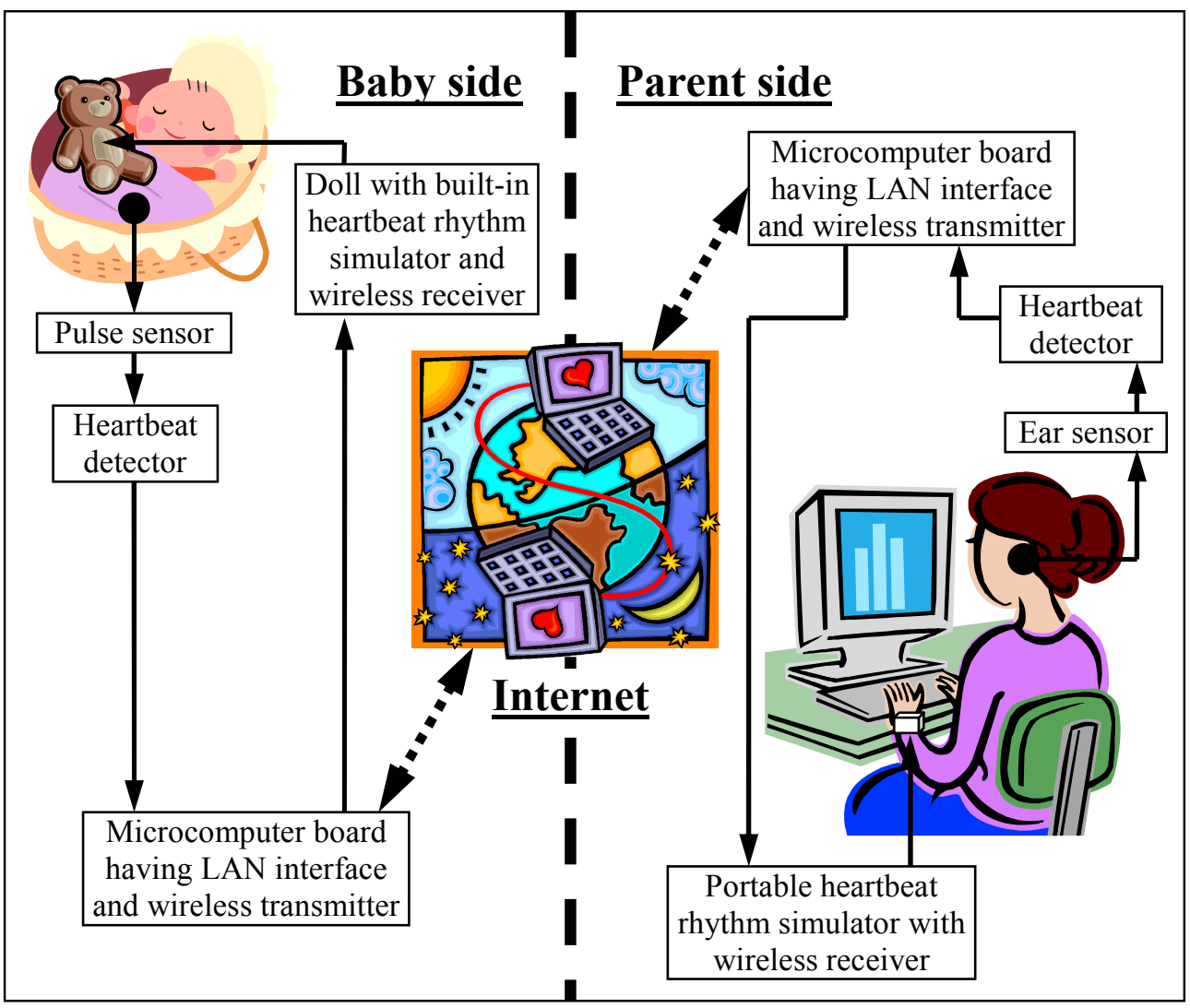

Fig. 1 Illustration of the proposed system for Internet transmission of the heartbeat rhythm

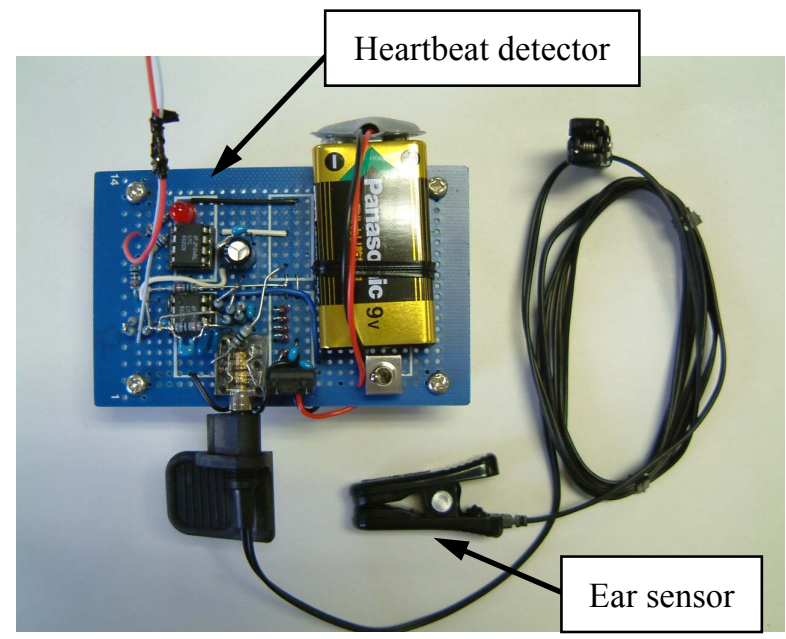

Fig. 2 Photograph of the ear sensor and the heartbeat detector 


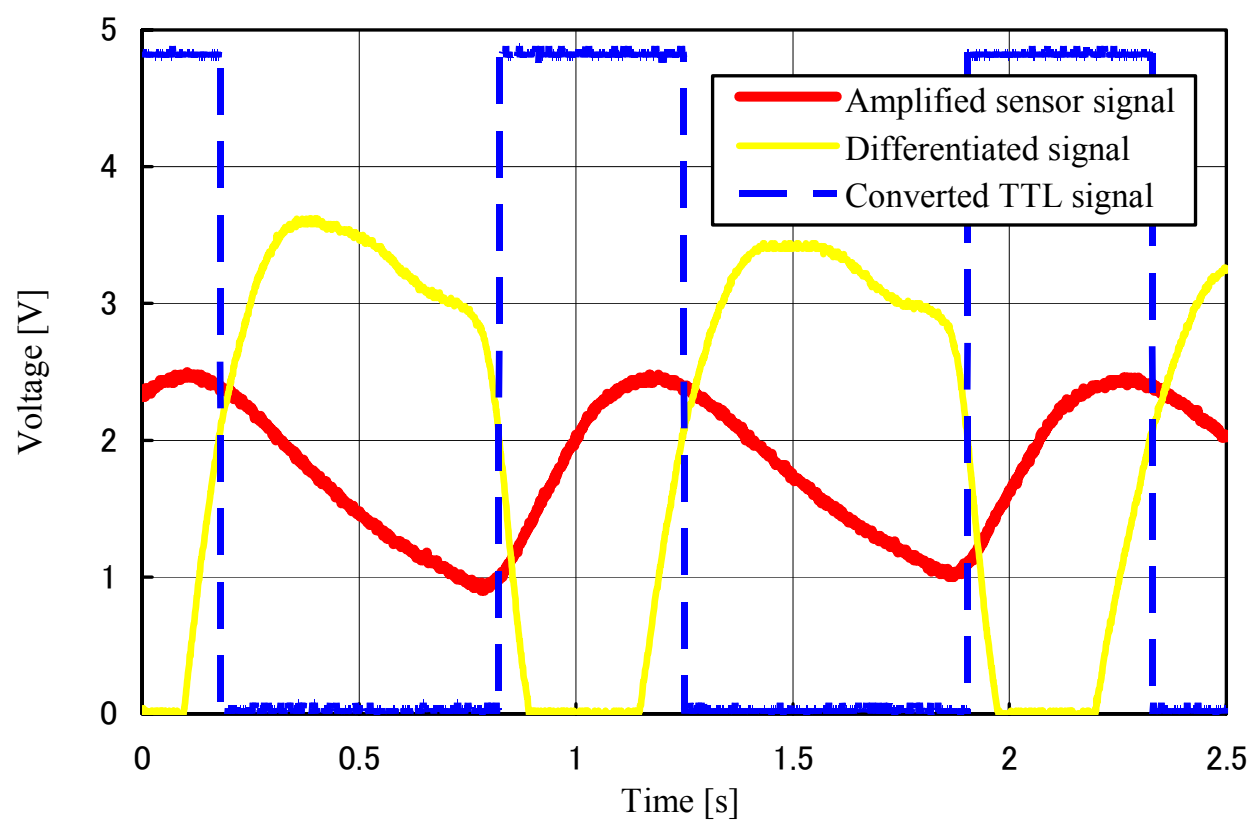

Fig. 3 Signals in the heartbeat detector

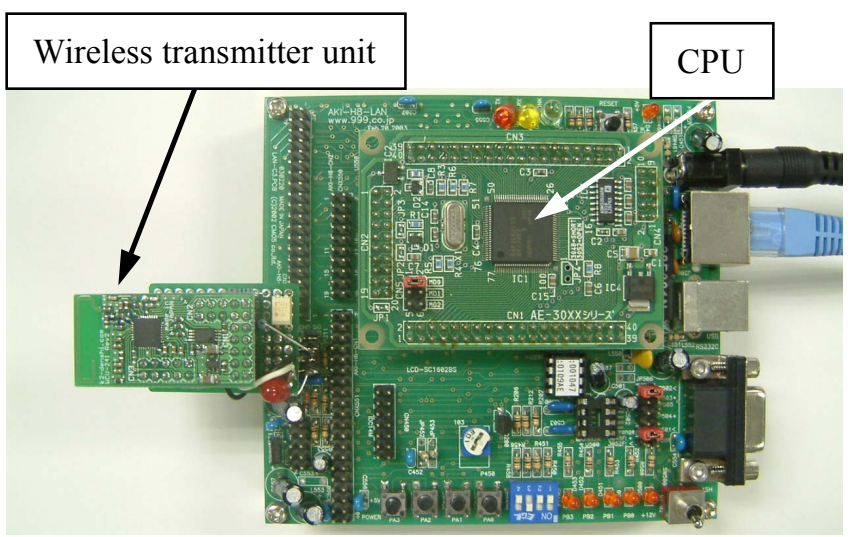

Fig. 4 Photograph of the single-board microcomputer having LAN interface with the small wireless transmitter

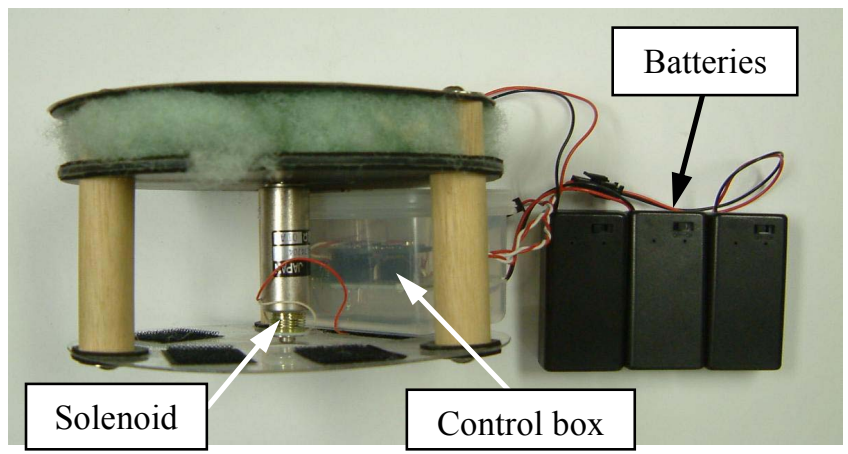

Fig. 5 Photograph of the heartbeat rhythm simulator 


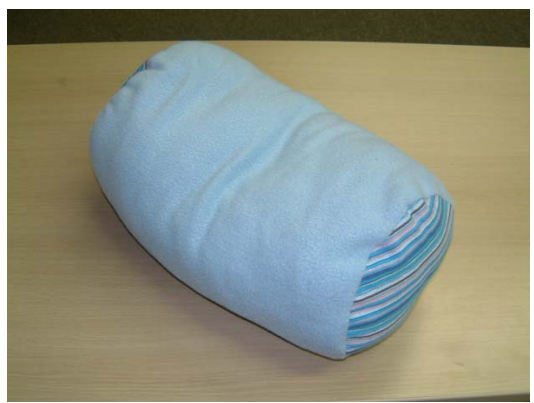

Fig. 6 Photograph of the pillow with the built-in heartbeat rhythm simulator

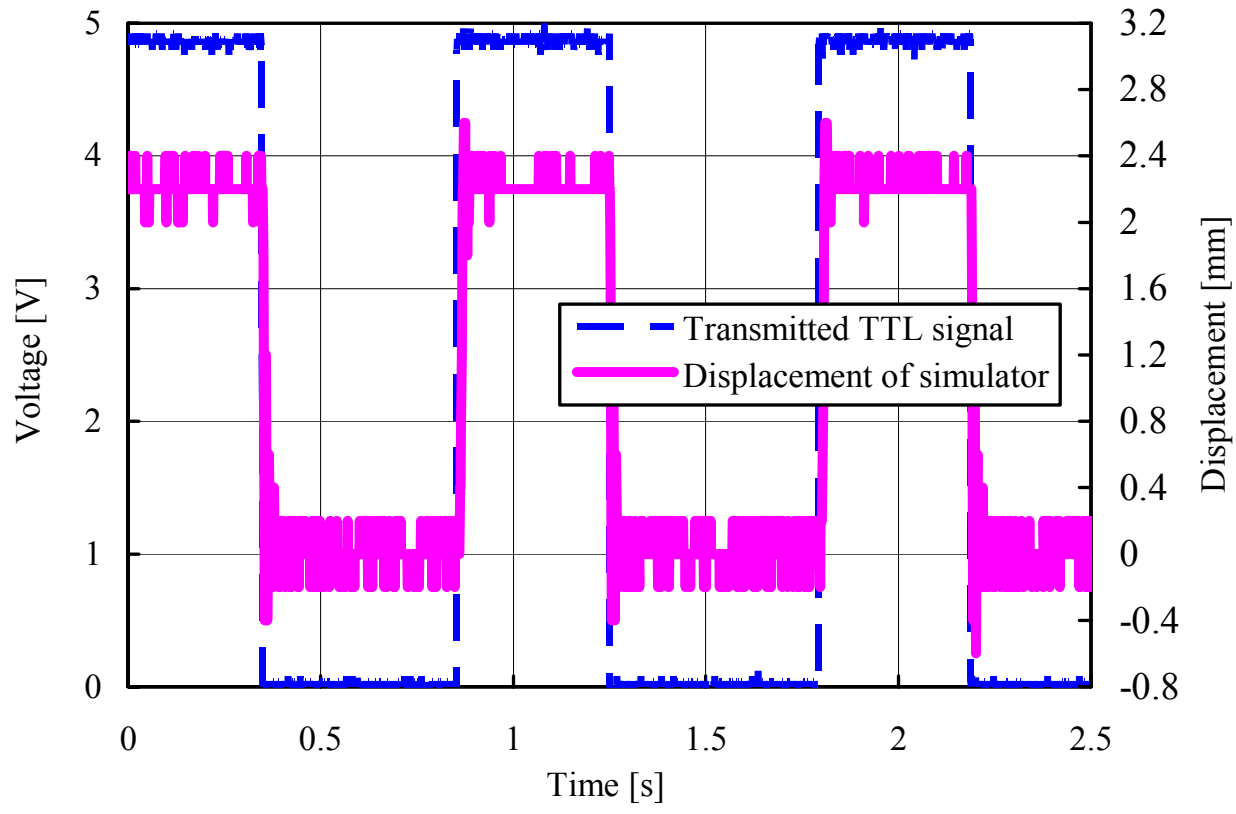

Fig. 7 Transmitted signal to the simulator and the displacement

The system architecture for transmitting the baby's heartbeat rhythm to the parent is the same as that for transmitting the parent's heartbeat rhythm to the baby. A pulse sensor is used for the baby, and a heartbeat rhythm simulator is used for the parent. Figure 8 shows the experimental model of the pulse sensor and the heartbeat detector used for a baby. The sensor is fixed on the baby's breast, under the clothes, by means of a rubber band in order to prevent its removal by the baby due to dislike. Figure 9 shows the portable heartbeat rhythm simulator, which is worn like a wristwatch by the parent and does not cause discomfort to the parent when he/she is working. The stimulation that most closely resembles the baby's heartbeat rhythm is generated by a solenoid by gently pressing against the arm skin, thereby simulating the pressure sensation as the blood is pumped. In this study, a simulator, in which the stimulation is generated by a vibrating motor, is used for miniaturization. Although the miniaturization caused a slight decrease in the resemblance of the simulated rhythm with the actual heartbeat rhythm, it was possible to keep the stimulator size small (40 mm (length) $\times 40 \mathrm{~mm}$ (width) $\times 15 \mathrm{~mm}$ (thickness)). The simulator is placed in a cloth bag and worn on the wrist by using a rubber band. 


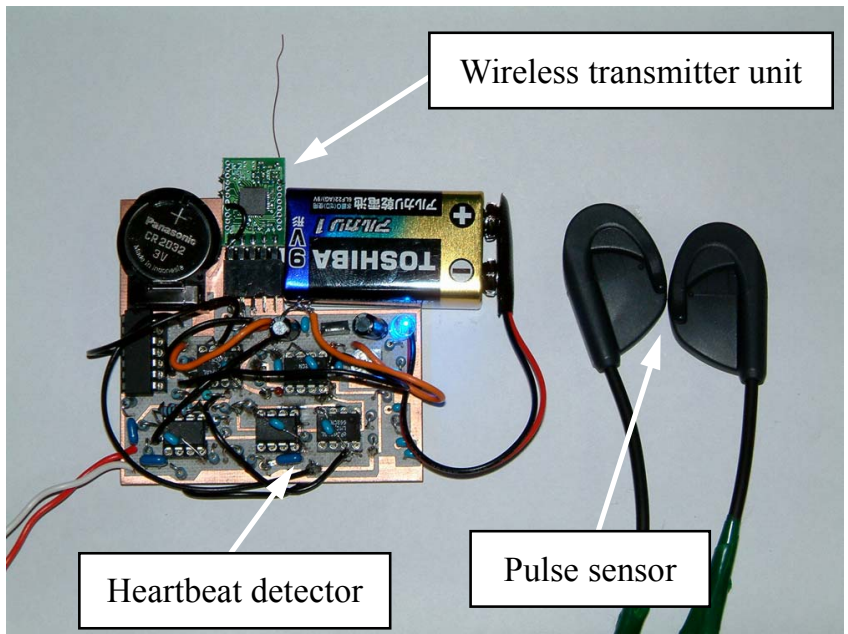

Fig. 8 Photograph of the experimental model of the pulse sensor and the heartbeat detector for a baby

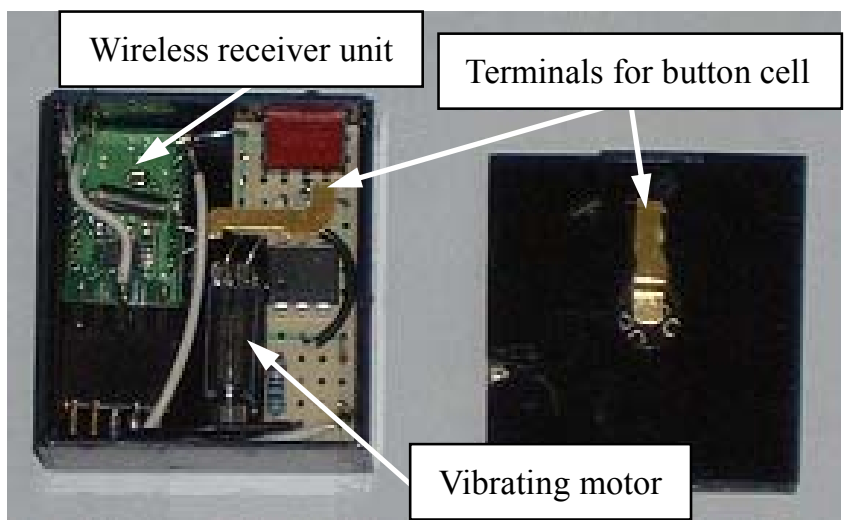

Fig. 9 Photograph of the portable heartbeat rhythm simulator worn like a wristwatch by a parent

\section{Reaction of Baby to Mother's Heartbeat Rhythm}

In order to confirm that the proposed system afforded comfort and peace of mind to a baby, an evaluation experiment was carried out. In the experiment, it was examined as to how a baby reacts to the transmitted heartbeat rhythm of its parent. Six families participated with their babies, and nine cases were evaluated: a 2-year-old female baby (A), a 5-year-old female baby (B), a 2-year-old male baby $(\mathrm{C} 1, \mathrm{C} 2$ : response was evaluated twice with an interval of two months), a 1-year-old female baby (D1, D2: response was evaluated twice with the interval of three months), a 3-year-old female baby (E1, E2: response was evaluated twice with an interval of five months), and an 11-month-old female baby (F).

\subsection{Evaluation Method}

The evaluation experiment was carried out in the living room of the baby's residence in cooperation with a person to whom the baby was well accustomed (for example, the baby's grandmother or aunt). By placing the equipment in a safe position and covering it with a cardboard box in order to hide it, the room was kept to the condition that the baby always played. Then, a video camera was set in such a way that the baby would always be in the 
picture frame, even if it moved. The baby's reaction during the experiment was recorded. The baby's mother was seated in a relaxed condition on a chair placed near a wall in the room and wore an ear sensor on during the experiment. Then, she was asked to respond to a questionnaire by observing the baby's reaction. In addition, the person to whom the baby was accustomed was asked to play with the baby so that the baby's attention would not turn to its mother.

For the first three families (A, B, and C), the heartbeat rhythm of the mother was simulated in a pillow placed in the baby's playing area for about $15 \mathrm{~min}$. Next, a switch installed on the single-board microcomputer was operated in such a manner that the baby did not notice; by this operation, the simulation of the heartbeat rhythm was stopped for about $10 \mathrm{~min}$ (the non-stimulation condition). Then, the heartbeat rhythm of the mother was simulated again for about $10 \mathrm{~min}$. Finally, an artificial heartbeat rhythm with a constant period, corresponding to a pulse of 80 , was generated by a function generator for about 10 min. The mother was asked to observe the behavior of the baby during the experiment and respond to a simple questionnaire before starting the experiment, after simulating each rhythm, and after finishing the experiment. In addition, she was asked to rank the rhythms in the order of their effectiveness in making the baby feel comfortable. The experiment was started after one hour of setting up the equipment in order to accustom the baby to the environment with the equipment. In addition, the presentation of the first rhythm was prolonged for about $5 \mathrm{~min}$ in order to accustom the baby to the experiment. And, the rhythm was changed to the next one if the baby seemed tired of the presented rhythm.

In order to evaluate the baby's condition, two types of questionnaires were prepared. In these questionnaires, some crossbars with five scales were placed at regular intervals, and epithet pairs, which were mutually contradictory, were typed at each end of the crossbars. Then, a circle was drawn as a sign by the baby's mother on the scale at the degree that was most true for each epithet pair as an impression of the baby's reaction. For the questionnaire used before starting and after finishing the experiment, the following four epithet pairs were selected: "Baby in a good mood - Baby in a bad mood," "Relaxed condition - Nervous condition," "Composed condition - Excited condition," and "Sleepy condition - Active condition." For the questionnaire used after presenting each rhythm, three epithet pairs were selected: "Easy feeling - Anxious feeling," "Like the condition - Dislike the condition," and "Enjoying feeling - Feeling without interest." Thus, the questionnaires were easy and could therefore be answered in about 10-20 s.

For the last three families (D, E, and F), the same experiment was carried out by changing the presentation order. The simulated artificial heartbeat rhythm was presented first followed by the simulated heartbeat rhythm of the mother, the non-stimulation condition, and again, the simulated heartbeat rhythm of the mother.

\subsection{Results and Discussion}

Table 1 summarizes the rankings of the presented rhythms in the order of their effectiveness in making a baby feel comfortable, as evaluated by the baby's mother.

It should be noted that the results shown in Table 1 do not represent objective evaluation, but the relative ranking evaluated by a baby's mother through the observation includes cases in which the baby did not show interest in the pillow that simulated rhythms and in which the baby was tired of the experiment. The average rank (presented in the last row of Table 1) indicates that as judged by a baby's mother, the simulation conditions can be stated in the order of their effectiveness in making a baby feel comfortable as follows: the second simulation of the mother's heartbeat rhythm, the first simulation of the mother's heartbeat rhythm, the simulation of the artificial heartbeat rhythm with a constant period, and the non-stimulation condition. In the first four cases (A, B, C1, and C2), the artificial heartbeat rhythm and the non-stimulation condition were not evaluated. In the last five 
cases in which the presentation order of the simulation was changed, only the non-stimulation condition was not evaluated, and the rhythm presented after the non-stimulation condition was evaluated high. Consequently, it is clarified that a baby tends to prefer the stimulated condition, particularly the heartbeat rhythm of its mother.

Table 1 Rankings of the presented rhythms: listed in the order of their effectiveness in making a baby feel comfortable, as evaluated by the baby's mother

\begin{tabular}{|c|c|c|c|c|c|}
\hline & \multicolumn{4}{|c|}{ Type of pulse } \\
\hline & & $\begin{array}{l}\text { Mother's } \\
\text { (1) }\end{array}$ & Non-pulse & $\begin{array}{l}\text { Mother's } \\
\text { (2) }\end{array}$ & Artificial \\
\hline \multirow{4}{*}{ 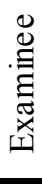 } & A & 2 & 3 & 1 & 4 \\
\hline & B & 1 & 4 & 2 & 3 \\
\hline & $\mathrm{C} 1$ & 3 & 4 & 1 & 2 \\
\hline & $\mathrm{C} 2$ & 1 & 3 & 2 & 4 \\
\hline \multicolumn{2}{|c|}{ Average } & 1.75 & 3.5 & 1.5 & 3.25 \\
\hline & & Artificial & $\begin{array}{l}\text { Mother's } \\
\text { (1) }\end{array}$ & Non-pulse & $\begin{array}{l}\text { Mother's } \\
\text { (2) }\end{array}$ \\
\hline \multirow{5}{*}{ 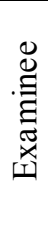 } & D1 & 2 & 1 & 4 & 3 \\
\hline & $\mathrm{D} 2$ & 2 & 3 & 4 & 1 \\
\hline & E1 & 4 & 2 & 3 & 1 \\
\hline & E2 & 1 & 2 & 4 & 3 \\
\hline & $\mathrm{F}$ & 2 & 4 & 3 & 1 \\
\hline \multicolumn{2}{|c|}{ Average } & 2.2 & 2.4 & 3.6 & 1.8 \\
\hline & $\begin{array}{l}\text { Mother's } \\
\text { (2) }\end{array}$ & $\begin{array}{l}\text { Mother's } \\
\text { (1) }\end{array}$ & Artificial & Non-pulse \\
\hline \multicolumn{2}{|c|}{ Total average } & 1.67 & 2.11 & 2.67 & 3.56 \\
\hline
\end{tabular}

Figure 10 shows the averages obtained from the responses to the questionnaire, i.e., the baby conditions observed by its mother after each rhythm was presented. It is clarified that a baby felt easy and liked and enjoyed the condition in which the heartbeat rhythm of its mother was simulated, rather than the non-stimulation condition or the condition in which the artificial heartbeat rhythm was simulated. However, with regard to the averages for the last five cases, the actual results obtained for the condition in which the artificial heartbeat rhythm was simulated are almost the same as those obtained for the condition in which the heartbeat rhythm of its mother was simulated.

Figure 11 shows the averages obtained from the responses to the questionnaire, i.e., the baby conditions observed by its mother before the experiment was started and after the experiment was over. It is clarified that the conditions before starting the experiment is almost identical to that after finishing the experiment, although there were cases in which the baby became sleepy or active.

Next, in order to evaluate the difference of a baby's reaction between the condition in which the heartbeat rhythm of its mother was simulated and the condition in which the artificial heartbeat rhythm was simulated, the evaluation experiment was carried out in cooperation with three families $(\mathrm{D}, \mathrm{E}$, and F). In the experiment, the heartbeat rhythm of the mother and the artificial heartbeat rhythm were presented simultaneously by using two heartbeat rhythm simulators. The evaluation was performed twice for each family.

The results showed that, except for one case, a baby's mother judged that the baby 
preferred the heartbeat rhythm of the mother rather than the artificial heartbeat rhythm, although the mother was not informed about the type of the rhythm simulated in each simulator.

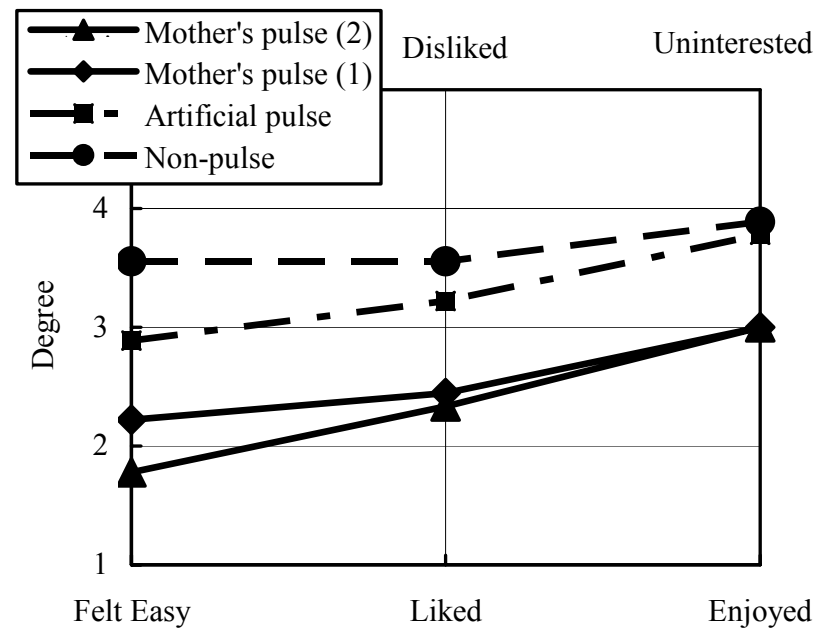

Fig. 10 Baby conditions observed by the mother after each rhythm was presented (Averages)

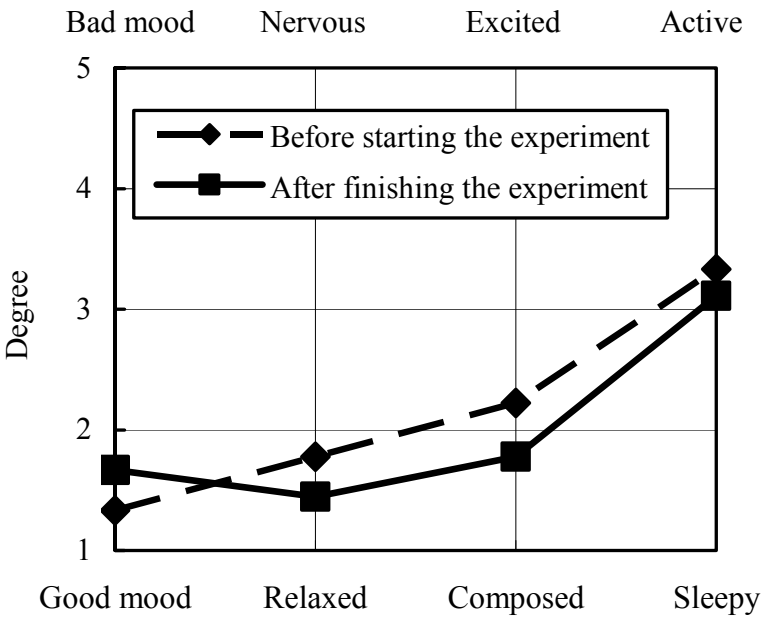

Fig. 11 Baby conditions observed by the mother before the experiment was started and after the experiment was over (Averages)

Then, in order to evaluate the difference of a baby's reaction between the condition in which the heartbeat rhythm of its mother was simulated and the condition in which the heartbeat rhythm of the person to whom the baby accustomed, the evaluation experiment was carried out in cooperation with three families (D, E, and F) and a new family. In the experiment, the heartbeat rhythm of the mother and that of the accustomed person were presented simultaneously or alternately by using two heartbeat rhythm simulators.

The results revealed that the mother could not recognize the difference of the baby's reaction between the two conditions.

From these results, it is confirmed that a baby tends to prefer the condition in which a heartbeat rhythm is simulated rather than the non-stimulation condition. Further, in the case 
that the baby is interested in the simulated sound, it tends to prefer the heartbeat rhythm of its mother or that of a person to whom it is accustomed rather than the artificial heartbeat rhythm with a constant period. Therefore, it is clarified that the proposed system can give comfort and peace of mind to a baby by transmitting the heartbeat rhythm of its mother.

\section{Effectiveness of Heartbeat Rhythm Transmission from Baby to Parent}

In order to confirm that the proposed system offers comfort and peace of mind to a baby's parent by transmitting the baby's heartbeat rhythm to the parent, an evaluation experiment was carried out in cooperation with two families with a 1 -year-old male baby and a 1-year-old female baby.

\subsection{Evaluation Method}

The evaluation experiment was carried out in the living room of a baby's residence in cooperation with a person to whom the baby was accustomed. A pulse sensor was placed on the baby's foot, and the accustomed person was asked to hold or cradle the baby so that the baby was prevented from moving too freely and being entangled in the wires of the sensor. The mother was asked to wear a portable heartbeat rhythm simulator on the wrist, and do some work in the kitchen. Then, the baby's heartbeat rhythm was simulated in the portable simulator for about $1 \mathrm{~min}$. Next, the artificial heartbeat rhythm with a constant period (corresponding to the pulse of 120) was simulated. Thereafter, the mother was asked to answer the question of whether she could recognize the difference between the two simulated rhythms. For the safety of the baby, a pulse sensor often used in a hospital and intended for a baby was used instead of the developed pulse sensor shown in Fig. 8.

\subsection{Results and Discussion}

Both mothers answered that they could recognize the difference between the heartbeat rhythm of their babies and the artificial heartbeat rhythm. Therefore, it is confirmed that the proposed system can indicate a baby's condition to its mother by transmitting the baby's heartbeat rhythm to the mother. Further, the mothers told us that they were anxious that their babies might get entangled in the sensor wires and put the sensor in their mouth. Therefore, it is necessary to develop a wireless pulse sensor that can be placed on the baby's breast, under the clothes, as shown in Fig. 8. In addition, we also received a suggestion that the proposed system was suitable for the nursing of a bedridden aged person.

\section{Conclusions}

In order to avoid a decrease in the birthrate, it is essential to develop social surroundings in which parents with a baby can work comfortably. Therefore, to provide comfort and peace of mind to a separated baby and its working parents, a system is proposed that transmits the parent's heartbeat rhythm to the baby through the Internet and vice versa.

The effectiveness of the proposed system was confirmed by conducting evaluation experiments. The experimental results revealed that when a baby is interested in a simulated sound, it tends to prefer the heartbeat rhythm of its mother or the person to whom it is accustomed rather than an artificial heartbeat rhythm with a constant period or the non-stimulation condition. The results also indicated that a baby's mother can distinguish the heartbeat rhythm of her baby from an artificial heartbeat rhythm by using the portable heartbeat rhythm simulator like a wristwatch. Thus, it was demonstrated that the proposed system can give comfort and peace of mind to both a baby and its parent. 


\section{Acknowledgement}

I thank the mothers and their babies for their participation and cooperation in the experiments.

\section{Postscript}

Before conducting the experiments, the participating mothers were informed by a letter about the aim of the experiments, the methods used, and the protection of private information, and they was asked to sign a consent form expressing their free will to participate in the experiments.

\section{Reference}

(1) KAWASHIMA, T., Development of Active Rocking Chair with Changing Swing According to Heartbeat Fluctuation, JSME International Journal, Series C, Vol.49, No.3,(2006), pp.814-820. 